\title{
Entre liturgie et incursions scéniques. Quand la chanson prend la parole
}

Between Liturgy and Theatrical Incursions: When Song Speaks

Joëlle Deniot

\section{(2) OpenEdition}

Journals

Édition électronique

URL : https://journals.openedition.org/rsl/1910

DOI : $10.4000 /$ rsl. 1910

ISSN : 2271-6246

Éditeur

Éditions Rue d'Ulm

Référence électronique

Joëlle Deniot, «Entre liturgie et incursions scéniques. Quand la chanson prend la parole », Revue Sciences/Lettres [En ligne], 6 | 2019, mis en ligne le 10 décembre 2018, consulté le 30 juillet 2021. URL : http://journals.openedition.org/rsl/1910; DOI : https://doi.org/10.4000/rsl.1910

Ce document a été généré automatiquement le 30 juillet 2021.

(C) Revue Sciences/Lettres 


\title{
Entre liturgie et incursions scéniques. Quand la chanson prend la parole
}

\author{
Between Liturgy and Theatrical Incursions: When Song Speaks
}

Joëlle Deniot

1 Édith Piaf, Germaine Montero, Rosy Varte: trois femmes proches en matière de génération, mais distantes du point de vue des époques traversées - Édith Piaf meurt en 1963, Rosy Varte, de huit ans sa cadette, s'éteint en 2012. Trois femmes qui laissent leur nom dans le monde de l'art et du spectacle ; trois femmes qui chantent, osent « donner de la voix » d'une façon engageant une certaine théâtralité, mais dans des registres socio-esthétiques fort éloignés. L'une ne s'essaie qu'une seule fois au jeu théâtral proprement dit $^{1}$; en revanche elle acquiert dans la chanson une réputation telle qu'elle devient le symbole pérenne de son âge d'or : c'est Édith Piaf. Germaine Montero se définit d'abord comme actrice. Menant parallèlement une carrière de chanteuse, lorsqu'elle incarne Mère Courage, elle s'appuie brillamment sur son double talent d'interprète. Rosy Varte, connue comme actrice au théâtre, au cinéma et à la télévision, mais qui a commencé par chanter dans le cadre du cabaret, impose sa voix dans les épisodes musicaux d'Ubu.

2 In fine, peu de ressemblances entre ces trois interprètes. Il faut renoncer à toute approche transversale. Si la mise en parallèle comparative s'avère si délicate à théoriser, c'est que les voix chantées de la scène théâtrale et les voix chantées de la scène chansonnière ne sont pas contemporaines. Entre le théâtre de la chanson et les chansons du théâtre, il existe bien des décalages d'histoire, de sens et de langue. On constate cependant des influences mutuelles dès la fin du xIXe siècle, avec les mutations $\mathrm{du}$ caf'conc', et surtout dès le premier tiers du $\mathrm{xx}^{\mathrm{e}}$ siècle. Notre approche concerne une parenté plus profonde. Les chansons relevant de la forme sanctuarisée du récital et les chansons inscrites dans l'univers dramatique ont peut-être pour point commun d'être les traces conservatoires de mondes qui s'éloignent: celui de l'effusion populaire 
partagée, celui de la mise à distance, au moins ponctuelle, des contradictions sociales. Au centre de la réflexion, l'idée de l'autonomie du geste musical.

\section{Le temps des chansons}

3 Pour parler de théâtre de la chanson, il faut une scène d'où l'on puisse la percevoir comme un nouveau territoire langagier, voire littéraire. Or la chanson - comme genre spécifique d'articulation de la musique et du verbe - n'acquiert ce statut que dans la modernité du début du $\mathrm{xx}^{\mathrm{e}}$ siècle. Si chanter est un anthropos immémorial, le temps des chansons dont on savoure la théâtralité est bien court. "C'est en observant MounetSully jouant Æedipe Roi que j'ai eu la révélation d'un cri possible, dans ma chanson : La Soularde », écrit Yvette Guilbert, célèbre diseuse du caf'conc' à la fin des années $1920^{2}$. Cette référence marque un tournant dans l'histoire de la chanson: son entrée dans une composition expressive à double dimension, la dimension vocale et la dimension plastique.

4 Selon ce que nous en savons, ni les caveaux, goguettes, sociétés ou cafés chantants, ni les diverses formes de transmission diffuse du répertoire populaire ne mettent en avant l'imagination visuelle du chanter. Les chansons appartiennent au domaine de l'oralité dynamique, celle qui accompagne les fêtes, les prières, les révoltes, les grands rites ; celle qui redonne souffle aux actions et symboles collectifs. Ce n'est que sous le Second Empire, avec le caf'conc', que la chanson commence à entrer modestement dans l'ère du spectacle. Modestement, car il ne s'agit que de quelques inserts burlesques où l'artiste a du mal à s'imposer. Un nouveau divertissement consistant à venir «voir chanter " les airs à la mode a bien fait son apparition, mais la posture d'attention requise au théâtre n'est pas ici de mise.

5 Au-delà de tout ce que l'on connaît sur les difficultés du passage à l'écoute silencieuse pour bien des arts scéniques, on ne peut s'abstraire de l'idée que pour la chanson quels que fussent la situation et le milieu social concernés -, il s'agissait toujours de se mettre en mouvement. Chansons de banquets plutôt bourgeois des caveaux, chansons politiques, chansons à boire des goguettes ouvrières, complaintes et romances populaires des chanteurs de rues: toutes mobilisent une adhésion active, une implication de cœur et de corps. Dans cette culture historique partagée, il n'y a pas de place pour une réception passive. Or la chanson-spectacle a besoin d'auditeursspectateurs. Il convient donc de s'acculturer à une réception plus distante, momentanément retenue.

6 Souligner ce passé, voire ce refoulé culturel des chansons, c'est montrer l'écart de sensibilité et de temps existant entre la création d'un espace propre à un entendre-voir de la chanson et la didactique pionnière d'Yvette Guilbert sur ce nouvel art de chanter une chanson. C'est aussi dire que sur la période dite réaliste et néoréaliste qui constitue l'épicentre de nos recherches, la chanson avant Piaf, puis avec Piaf, occupe une sorte d'entre-deux. Elle est bien sûr un spectacle parvenu à son stade de maturité interprétative ; toutefois, cet art reste encore une «poétique sociale ${ }^{3}$ » dont le désir, la mémoire, l'expérience font partie des décors et ambiances familiales.

7 Yvette Guilbert et Édith Piaf déclarent à quelque trente années de distance que, pour chanter en lien avec soi, avec le monde, il faut beaucoup observer. Toutefois, si l'une écrit qu'elle trouve matière à styliser ses personnages à travers l'esprit des portraits, le 
jeu des acteurs, la médiation de tous les beaux-arts, l'autre suggère à demi-mots ne s'inspirer que de rencontres plus directes, plus vives avec le réel. Quoi qu'il en soit, Piaf et ses devancières donneront à la chanson un tour interprétatif inédit. Certains à leur propos parlent de "théâtre expressionniste ». La formule a le mérite de pointer l'idée d'une double rupture: celle de la chanson devenue art scénique et celle de cet art scénique ébauchant d'autres perspectives pour la représentation théâtrale. C'est grâce à cette mutation esthétique de l'objet-chanson à laquelle Piaf a contribué que se réalise la confrontation et/ou l'alliance de l'expression chansonnière (des partitions de petits formats, de la langue vernaculaire commune ${ }^{4}$ ) avec la tradition théâtrale, comme le dessinent les exemples de Germaine Montero et de Rosy Varte.

\section{De l'oreille à l'esprit : horizons d'écoutes}

Oubli des archives sonores des représentations théâtrales dans la théâtrologie contemporaine. Oubli de l'oralité et plus encore de la vocalité du côté des sciences sociales. En chacun des contextes de recherche où nous nous situons, nous sommes affrontée à une durable hiérarchisation des connaissances. La perception visuelle relayée par les sciences et techniques de l'optique est considérée comme notre sens le plus analytique, le plus proche du concept. Nous évoluons dans une intellectualité de géomètre plus ou moins taraudé par un imaginaire de voyant.

\section{Aesthesis familière contre analyse formelle}

Devant le silence sociologique, les travaux d'ethnomusicologie orientèrent nos premières tentatives d'écoute raisonnée des chansons. Toutefois l'ethnomusicologie ou la musicologie ne se penchaient que sur des partitions, des énoncés, des formes retranscrites, coupées de la situation de communication, autrement dit sur des traces sans corps ni voix. Les succès d'une sémiologie générale ${ }^{5}$ fortement présente dès les années 1960 encourageaient cette lecture musicolinguistique qui s'efforçait de concevoir la chanson (ou le chant) comme l'articulation entre deux systèmes sémiotiques comparables (sonores et séquentiels) : l'un marqué par l'unité minimale du phonème et les segmentations phonologiques, l'autre par l'unité solfégique et les organisations harmoniques, la prosodie du mètre poétique imposant toujours sa loi dans la mélodie française ${ }^{6}$ du moins - aux arrangements du rythme musical. Le modèle d'analyse structurale de la langue régnait alors sur toutes les interprétations en sciences humaines. Ce chef-d'œuvre de réduction analytique de l'acte langagier mettait ipso facto la chanson hors énonciation, hors écoute ${ }^{7}$. Car écouter une chanson ce n'est pas saisir de façon plus ou moins articulée deux registres de langages et de sons, c'est ressentir une vibration de significations confusément mêlées.

Fallait-il alors, pour retrouver la chanson, se rapprocher de l'écoute familière? Se détacher de toutes ces analyses formelles si éloignées de l'expérience esthétique immédiate? Afin de réentendre la chanson vivante, éphémère, incarnée, ne convenaitil pas de faire confiance au sens commun de cette musique-verbe-voix indissociable, de retrouver une intelligence de l'intuition? 


\section{Avènement de la centralité vocale}

11 Entendre la chanson, entendre un répertoire, un(e) interprète : est-ce aussi simple qu'une première impression le laisse supposer? Non, parce qu'entendre en compréhension est une sorte d'opération de reconstitution. Et si rien n'est anhistorique dans la chanson, le lien entre une chanson et la singularité d'une voix, qui semble actuellement évident, requiert lui aussi quantité de métamorphoses. Certes cette question du corps vocal (justesse, débit, force) est inhérente à toute participation à l'acte de chanter en solo ou en chœur. On retrouve dans les collectes des folkloristes du dernier tiers $\mathrm{du} \mathrm{XIX}^{\mathrm{e}}$ siècle des annotations à propos des voix requises pour telle ou telle chanson $^{8}$. Mais c'est autre chose de lier empreinte d'une voix et esthétique d'un chant, signature vocale et titre de chanson, irréversiblement scellés en objet de mémoire. Ce déplacement de l'horizon d'écoute et de sensibilité à l'expressivité, à l'esthétique vocale, suppose :

- l'émergence de la singularité mélodique. De la fin de la Renaissance jusqu'au milieu $\mathrm{du} \mathrm{XIX}^{\mathrm{e}}$ siècle prévalent les timbres ou fredons, c'est-à-dire l'interchangeabilité des paroles sur des airs connus. À une chanson correspondra bientôt une mélodie: Béranger initie cette mutation artistique de la chanson;

- l'émergence - sur le modèle de la dramaturgie - d'un travail du phrasé interprétatif. Nous l'avons souligné d'entrée avec Yvette Guilbert. Toutefois, pour Yvette Guilbert, la persona scénique du chanteur-interprète n'a aucun lien avec la personne qui chante. C'est le détachement du jeu qui est l'objet de sa quête. Si elle conçoit bien l'art interprétatif dans toute sa dimension plastique, ce n'est pas elle qui inaugure l'ère du rapprochement entre personnage et personne dans l'univers de la chanson. Or ceci est décisif pour la focalisation sur la voix et sa singularité substantielle dans ce tout organique du texte-musique-interprétation que va devenir la chanson, du moins dans la période qui nous occupe ;

- l'émergence du sujet dans la voix des chansons, permettant à cette forme musicale d'accéder pour un temps à son lyrisme propre. Ce passage à la révélation explicite d'un «je» dans l'œuvre constitue un tournant dans toute pratique artistique. Dans la chanson, cette odyssée du sujet dans la voix suscite un climat d'empathie des passions, un mouvement d'identification entre artiste et auditeurs-spectateurs. Elle ne requiert pas seulement le support socio-esthétique de l'interprète (café-concert, SACEM, théâtralisation de la performance chantée) mais se greffe également sur d'autres logiques plus sociétales. C'est au moment où les qualificatifs "réaliste ", " sociale ", «vécue » apparaissent que la chanson amorce cette métamorphose émotionnelle. Art populaire, la chanson, pour peupler l'âme collective, requiert d'une part l'apparition, à la fin $\mathrm{du} \mathrm{XIX}^{\mathrm{e}}$ siècle, du peuple comme sujet historique par alliance du politique et du romantique; d'autre part l'invention d'une intimité du tragique social lié à la Commune, aux catastrophes et répressions ouvrières, à la Première Guerre mondiale, à l'entre-deux-guerres ;

- l'émergence d'artistes-prolétaires, susceptibles de porter un tel écho du monde. Il y aura des paroles d'hommes et des voix de femmes. On pense à Fréhel, à Damia, à Piaf.

Cette centralité de la voix expressive et personnalisée met également un point d'orgue au processus d'apparition de la chanson comme œuvre originale. Lorsque Germaine Montero ou Rosy Varte chantent, cette révolution esthétique est totalement accomplie. Toutefois, entre les trois artistes mises en parallèle, la perception de la signature vocale $^{9}$ va changer de portée et de sens: il n'en va pas de même pour une Piaf 
s'identifiant corps et âme à son chant, pour une Montero qui se partage entre performance d'actrice et enregistrement de répertoires chantés, et pour Rosy Varte occasionnellement chanteuse au service d'une mise en scène.

\section{Édith ou la conviction vocale}

13 Porter l'attention sur la vocalité ne donne pas ipso facto les clefs les plus pertinentes pour son écoute. En ce qui concerne Piaf, le principal obstacle réside dans l'extrême familiarité de son intonation. C'est tout l'intérêt sociologique d'une telle figure dont l'aura traverse tout le siècle. Mais il est impossible au chercheur d'être dans l'étonnement d'une première écoute. On l'entend sur fond d'imaginaire saturé d'habitudes, de récits préexistants. Ce qui est vrai de toute écoute l'est ici de façon particulièrement frappante.

\section{Écrire une voix}

Comment écrire une voix ? Les disciplines peu nombreuses qui tendent à retranscrire les voix les soumettent à la représentation graphique, les fragmentent en unités d'accentuations phonétiques, les réduisent à l'abstraction de la mesure, à l'épure du tableau bidimensionnel, aux données du sonagramme. Tout se passe comme si aussitôt captée, la voix devait s'effacer. Si la panoplie des graphes est infidèle à la voix, dans l'impossibilité d'en retenir les rumeurs expressives, que peut le texte littéraire?

D'abord nous sommes confrontés à l'encerclement des métaphores visuelles. Pour la voix, on parle de couleur, de paysage, d'ornements, de profondeur, de hauteur, d'ambitus (lat. pourtour), de strates: images de peintre, d'architecte, de géographe. Mais on parle aussi de rythme, de tessiture, de timbre, de grain, d'âpreté, de légèreté, de souffle, de cri, de chuchotement, de voix d'or et de velours: images parfois plus sonores et mêlant surtout perceptions auditives et tactiles. Finalement les métaphores les plus usuelles de la voix nous renvoient à un registre kinesthésique engageant sensations croisées de l'œil, de l'ouïe, de la peau. C'est sans doute une chance qui s'offre pour accéder au verbe de la voix.

On sait que Piaf a suscité de nombreuses publications, surtout des biographies, comme si écrire sur elle, c'était nécessairement décrire son parcours qui se prête si bien, il est vrai, à la structure narrative : l'enfance vagabonde, les amours multiples, les accidents, l'ascension, la chute, la vie hors norme en tout domaine. Mettre son chant au cœur du texte était un tout autre projet. En ce sens, la première chose qui s'impose en l'écoutant, c'est sa conviction vocale, terme qui relève plutôt de l'art oratoire. Cela n'est pas innocent car si l'on va toujours parler de Piaf comme "The Voice» de son époque et même au-delà, ce n'est pas du tout ce qu'elle est... dans la mesure où, dans son cas, auralité et oralité ne sont pas dissociables. La rage de chanter de Piaf, c'est la rage de porter haut aussi bien des paroles, des récits que des mélodies. Elle est femme d'un répertoire dont elle gardera les tonalités fondamentales de plaisir et de douleur tout au long de sa carrière. Un répertoire qui s'inscrit dans un tournant culturel des voix populaires : la venue à maturité d'un besoin de signifier l'être intime, nostalgique ou tragique.

17 C'est sur ce refoulement de l'intime tourment (fureur d'aimer, ruines du cœur), sur ce silence affectif, marque et honneur de l'ethos des familles ouvrières et populaires de ce 
temps, que ces chansons vont pouvoir passer la rampe. Piaf est dans cette filiation d'un dire spécifique de la voix prenant en charge l'indicible des spectateurs et auditeurs.

Écouter le langage signifiant du chant de Piaf ${ }^{10}$, c'était se défaire d'un ethnocentrisme intellectuel portant à penser que les mots et tournures trop simples de ces chansons-là n'ont pas la dignité d'un objet d'étude. Si les 300 chansons enregistrées par Piaf ne sont pas d'égale qualité, les plus bouleversantes sont pourtant d'une justesse de style exemplaire. En effet, qu'est-ce que l'esthétique d'une énonciation si ce n'est le plus grand ajustement possible d'une forme à un sens qu'elle oriente en retour? Or, que tente d'imposer le style du chant de Piaf ${ }^{11}$ ? Le tressaillement le plus direct, le plus charnel possible d'un état de vertige, de danger, de perte où les mots sont comme suspendus de leur assurance performative. Entendre la signifiance de son chant, c'est accueillir le fait de ces états de choc qui ne souffrent pas la distance d'une langue trop écrite ${ }^{12}$. Évacuer la préciosité, l'effet littéraire, c'est précisément se maintenir dans la densité des affects, au bord de l'imprononçable.

D’une façon plus générale, élision, vibrato, mélismes, allongement syllabique du glissando, alternance entre notes chantées et silences vocaux, points de suspension du simple la la la, extinction brutale du son... la chanson réalise de multiples sorties de route de la langue qui nous lient à l'instabilité, à l'inachèvement du sens. Sans aller jusqu'à l'exercice d'une découpe du phonème portant la signification hors les mots telle que la composition de Gainsbourg l'effectue sur le prénom Lætitia, Elaeudanla Teïtéia (1962), Piaf, dans un registre moins intellectualisé, passe bien souvent outre la raison de la langue. C'est à sa façon qu'elle nous mène implacablement au ras des sensations, usant à merveille de ce pouvoir consolant, affolant du chant porteur de nos nuits.

Écouter la signifiance de son chant, l'écrire, c'était aussi écouter tout l'univers des " chansons réalistes", des "chansons vécues " qui plante - sur schème narratif - un décor parcouru de figures typiques de l'errance, dessinant toute une topographie des villes. Il existe bien un imaginaire de la chanson réaliste, néoréaliste, que l'on peut décrire puisqu'il va prendre le pas sur toute la chanson populaire et que Piaf se nourrit de cette physique et de cette sémantique vocales ${ }^{13}$. Germaine Montero qui chantera Bruant puis Mac Orlan s'inscrira dans une ultime réappropriation littéraire de cette veine chansonnière.

\section{À double entente}

Piaf se situe au tournant décisif du passage technique à la chanson médiate. Fréhel, Damia, Marie Dubas tenaient leur public en émoi dans le face-à-face. Au gré des mutations de l'enregistrement discographique et radiophonique, Piaf est la première de cette lignée d'interprètes à faire passer autant de nuances et de pathétique dans la situation hors public que dans la situation de scène. Celle qui portera à son acmé le soin du geste est aussi celle qui, sans l'aide du voir de la voix, parviendra à imposer l'intensité d'une présence.

Toutefois, si tous les embrasements de son grain de voix perdurent dans l'enregistrement audio, conférant à la chanson médiate la même puissance communielle, les documents audiovisuels nous donnent aussi beaucoup à lire sur le langage scénique de l'artiste. Prenant une conscience progressive de la place de cet écouter-voir, de ce théâtre de la voix dans la chanson, prenant aussi conscience de la force de ce paralangage de l'incantation se réverbérant sur le visage, dans le 
mouvement des mains, dans les postures d'adresse à l'interlocuteur chez Piaf, nous décidâmes de considérer ces documents audiovisuels comme corpus et objets de travail. Cette mystique incarnée du chant, il fallait l'écouter, il fallait la voir. En regroupant les séquences gestuelles, c'est une autre écoute de ses chansons qui se révélait. Grâce à ces mises en rapport inattendues, une méthode de va-et-vient entre l'audible et le lisible se fit jour. Le Billard électrique (Louis Poterat/Charles Dumont, 1961) est par exemple une chanson très " cinématographique » : la cinétique mélodique, le staccato vocal et l'effet narratif de suspense cumulent leurs effets dramatiques pour souligner à l'extrême un précipité de l'attente. Piaf y est très convaincante et dans la symbolique du son et dans celle du mime. Ce travail sur le lisible de la traduction scénique a conforté notre audace à mettre des mots sur cette voix inouïe.

\section{D'une liturgie à l'autre}

Du point de vue de l'étymologie, le mot «liturgie » (du grec $\lambda \varepsilon i \tau o u p \gamma i ́ \alpha)$ désigne une fête ou un loisir payé par un mécène. Sous le regard anthropologique, il désigne tout acte cérémoniel public dédié à quelque divinité ou substitut sacralisé du monde profane. Sa forte dimension rituelle s'appuie sur des codes réglés par l'ascendance de la tradition. Sans doute pointe-t-il cette proximité fréquente du culturel et du cultuel que, par exemple, tout concert de musique, rock, folk ou lyrique, reproduit lors des grands rassemblements festivaliers.

Piaf se situe historiquement et personnellement dans la conquête de l'espace rituel du chant. Il va lui falloir passer par tout un chemin d'acculturation pour accéder à cette autorité liturgique de sa performance. Le parcours de Piaf ne repose que sur une soif et une ambition autodidactes. C'est donc à partir de son talent vocal «naturel » qu'elle doit tout fonder : la distance d'écoute entre elle et les spectateurs quand elle quitte la chanson des rues; l'accès au sens des mots, aux nuances de la langue des mélodies retenues; le saut dans la symbolique du spectacle englobant discipline du corps, du costume, de l'expressivité vocale et gestuelle.

Au gré de ces métamorphoses sociales, psychiques, langagières ${ }^{14}$, la forme du récital parachève l'acte cérémoniel de ce temps chanté. Il est actuellement difficile de visionner un récital complet de Piaf, les archives aussi bien sonores qu'audiovisuelles ne donnent à voir et à entendre que des montages aléatoires. Reste l'accès public (via Internet) à un concert entier filmé à Nimègue en 1962. Lors de cette prestation, elle enchaîne douze chansons dont dix récemment inscrites à son répertoire. Pour le final, elle reprend deux succès confirmés La Foule et Milord. Le rythme est intense. Tout son être est concentré.

Sans doute est-elle dans une urgence, on est en 1962; c'est sa dernière année d'apparition en public et les stigmates de la maladie sont visibles. Deux choses retiennent l'attention: d'abord une détermination de la volonté poussée à l'extrême dont les indices filtrent à travers ce contraste entre la déformation des mains, de la posture dorsale, de la bouche même, et la ferme tenue de la voix, du souffle, ainsi que de l'intensité interprétative. Une voix toutefois un peu trop tendue, au glissando un peu rude, une interprétation empreinte d'un masque trop hiératique. Sans doute souffre-telle. Mais elle semble aussi se mouvoir dans une sorte de transport chamanique où le chant cherche à prendre le pouvoir sur le corps : son statut d'icône est confirmé. Il n'est qu'à observer le public saisi par la caméra. En effet, second élément à souligner : limitée 
dans ses mouvements, elle ne peut souvent qu'esquisser la signature gestuelle de chacune de ses chansons. Mais l'ellipse de ce pas de danse au dernier couplet de $L a$ Foule, l'amorce de cette expressivité des mains, toute ébauche... suffit aux spectateurs gagnés au code narratif de son tour de chant. Même tracé en pointillé, le canevas symbolique de la chanson, de son mimodrame, est pleinement reçu par le peuple des croyants réunis autour de la messagère du culte d'Éros.

Si Piaf a dû fonder une liturgie dont le souvenir perdure et s'efface à la fois, la cérémonie théâtrale est quant à elle depuis fort longtemps entrée dans son âge classique, âge que désormais elle reproduit et perturbe sans cesse. La langue théâtrale connaît dès la première moitié $\mathrm{du} \mathrm{xx}^{\mathrm{e}}$ siècle une ère de rupture avec les traditions scéniques. Les chansons n'y occupent-elles plus qu'une place périphérique? Le plaisir de la chanson peut-il au contraire encore réserver des surprises dans cette économie bouleversée de la représentation théâtrale ? Nous examinerons cette question à travers les exemples de Mère Courage et de Mère Ubu.

\section{Germaine ou la voix au prisme du rôle}

Pour définir la personnalité vocale de Germaine Montero, il fallait aller au-delà des commentaires de presse louant sa prestation dans le rôle-titre de Mère Courage. Côté jardin, il y avait la reconnaissance publique d'un langage scénique bien maîtrisé ; côté cour, l'ombre de la comparaison avec le jeu d'Helene Weige ${ }^{15}$. L'aventure s'annonçait ainsi : comment se frayer un chemin de pensée, à côté ou à l'écart des débats entourant l'histoire de cette réception?

\section{Deux ou trois choses que je savais d'elle}

La voix de Germaine Montero s'était gravée en moi avec deux chansons mémorables : Les Enfants qui s'aiment de Prévert/Kosma et la Chanson de Margaret de Mac Orlan/ Marceau. Dans le chant de Prévert enregistré en 1952, l'intonation sombre et grave de Germaine Montero s'impose avec beaucoup d'assurance et d'éclat dans la montée chromatique. Les Enfants qui s'aiment, emblème mélodique des Portes de la nuit ${ }^{16}$, sera repris par des interprètes célèbres : Catherine Sauvage, Juliette Gréco ; les réécoutant, nous observons qu'elles suivent l'empreinte poétique donnée par Germaine Montero. Cette voix est donc suffisamment marquante pour faire norme, devenir source d'une culture interprétative.

L'attention portée à la Chanson de Margaret est plus directement rattachée à mon travail de longue durée sur les différentes déclinaisons de la « chanson réaliste ». Les chansons de Mac Orlan, entre réalisme et surréalisme, retiennent des souvenirs de lieux au sortir des villes, des ambiances de ports, de bars, de matelots en partance. On entre dans les années 1950, la romance noire de la fatalité n'est déjà plus vraiment dans l'air du temps, mais son charme continue d'opérer pour les auteurs et les interprètes. Mac Orlan compose dans ce sillage. Forme narrative, personnages archétypaux, tableaux naturalistes des marges sociales: l'esprit si ce n'est la lettre de la complainte faubourienne est bien là. La Chanson de Margaret est un classique de cette poésie réaliste tardive ou retrouvée. Germaine Montero, première interprète de Mac Orlan, imprime son sceau sur cette mélodie complexe. Timbre clair, souffle puissant, tempo vif: Mac Orlan parle d'un « secret Montero » dans l'appropriation de ce titre. 
31 Dans cet exemple de chant réaliste, pris au sens large, le style vocal de Montero s'apparente bien aux spécificités de jeu observées chez les chanteuses emblématiques du genre: une diction toujours nette détachant les intentions et les mots, une implication soutenue dans l'expression chantée, une aisance dans les graves, mais aussi un mélange de force vitale et de mélancolie si caractéristique d'une Fréhel par exemple. Outre l'écho du lyrisme littéraire qui l'habitait, cette voix était donc également rattachée à la rumeur et à la veine populaire la plus féconde de son temps. Mais il était troublant qu'à la différence d'une Damia, d'une Berthe Sylva, d'une Yvonne George, d'une Fréhel, d'une Piaf, le grain de voix de Germaine Montero ne fût pas reconnaissable dès les premières syllabes. Pourquoi ce léger effacement de l'identité vocale à l'entrée même dans le voyage mélodique ? Tout semblait indiquer qu'il fallait chercher sa singularité dans un tout autre espace.

\section{"Venue à la chanson avec ses dons de comédienne ${ }^{17}$ "}

C'est le hasard de la découverte d'une archive audiovisuelle qui me mit sur la piste. Silhouette fine, coiffure austère, port de tête aristocratique, Germaine Montero s'avance pour chanter $\mathrm{Du}$ gris $^{18}$, acmé de l'inspiration réaliste. Malgré l'amorce d'une intonation gouailleuse, le geste vocal est maniéré, la distance au rôle, palpable. Soudain tout bascule. Germaine Montero s'engage sans réserve, invente sa métamorphose au fil des refrains, va même jusqu'à souligner ce roulement des " $r$ ", vibration si familière des mélodies populaires, mais aussi présence trop inconvenante du corps dont le refoulement s'impose, et dans le verbe, et dans le chant, dès l'après-Seconde Guerre mondiale.

Voilà qui incite à se demander s'il ne faut pas, pour Germaine Montero, changer la perspective retenue pour analyser les grandes réalistes et Piaf, dont les voix, les chansons restent miroirs d'expériences subies: solitudes liées à la misère sociale, à l'écart des bonnes mœurs... Si cette incarnation de l'authentique ne les exempte pas d'un ensemble de façonnages expressifs permettant de les identifier comme artistes, reste à savoir ce qui est premier dans l'esthétique recherchée : le désir d'affirmation de l'émotion vitale que l'on creuse, que l'on traque, ou la volonté de s'inscrire dans le paysage de la référence dont on s'acculture, dont on se grandit. S'il faut, en effet, inverser la perspective pour Montero, c'est que celle-ci est d'abord formée par choix initial, par choix radical, à l'exercice théâtral de «l'imitation de l'action des hommes ${ }^{19}$ ", à la discipline de l'emprunt des masques et des rôles où le «je » se doit d'être l'Autre.

Germaine Heygel, née en 1909 à Paris, débute sur les planches à Madrid sous le nom de Montero et sous la direction de Federico García Lorca. De retour en France, elle joue dans des adaptations françaises du théâtre espagnol. Chantant des airs traditionnels pour un cercle d'amis, elle finit par rencontrer Agnès Capri qui l'enrôle dans son cabaret de la rue Molière. Elle s'y produira durant deux saisons dans son répertoire de chants castillans. Chanter en public est donc pour elle une sorte de hasard né de son métier de comédienne au centre de sa vie artistique jusqu'en 1953. À partir de cette date, chanson et théâtre retiennent à parts égales son investissement et son exigence. C'est à quarante ans, après une longue immersion dans le répertoire théâtral, que cet équilibre s'opère. Elle sera la seule interprète française, et cela pour longtemps, à disposer de ces deux gammes artistiques à hauteur équivalente de maîtrise et de succès. 

advenue via la scène hexagonale sous signature de l'art brut, subjectif et vital des chanteuses populaires. À l'opposé de l'incarnation aiguë du sentiment, le chant de Germaine Montero doit s'appréhender sous l'angle de la transposition d'un ressenti inéprouvé, sous l'angle d'une esthétisation mesurée, mais jamais ironique, du tragique populaire. Sans doute un aspect du « secret Montero » se loge-t-il dans un double refus : refus de l'imitation de l'original émoi et refus du second degré. La marge de jeu est délicate. On ne joue pas si aisément avec l'authentique. Mais si, comme chez Germaine Montero, l'authentique s'instille par touches et changements imperceptibles dans la marge des sensations émises, dans l'aura des tonalités et des mots, dans le dessin de leur déchirure ${ }^{20}$, vérité et vraisemblance finissent par se rejoindre. Point instable d'attention extrême, point enfoui de la sensibilité commune, atteint dans l'incorporation et le rite de la chanson. Si, dans son répertoire, les auteurs lettrés du "réalisme de la deuxième chance » sont dans le droit fil de son travail artistique, plus étonnants sont ses choix du grand chant flamenco, des chansons de barrière et des chansons du fatum social. Car ces genres s'adossent à une poésie cruciale, mélancolique de la voix, alchimie des rumeurs de la vie, au-delà même de l'enveloppe de son dire et de sa rengaine. Comme un chant derrière le chant. La référence à l'art de la comédienne est éclairante mais ne suffit pas. Il faut bien imaginer chez elle quelque complicité d'âme et de cœur avec cette douleur, cette chaleur vitale des exilés, des "gens de peu », lui permettant d'en emprunter les souffles.

Comment penser alors la personnalité vocale de Germaine Montero? Son unicité est nécessairement attachée à son phrasé, au déroulé temporel de la mélodie et du récit et non à son timbre, dont on a vu qu'il n'était pas reconnaissable dans l'instant, à la différence des chanteuses populaires admirées de l'entre-deux-guerres. C'est dans l'intervalle entre l'attaque de la chanson encore policée, presque neutralisée, et le déplié de son verbe mélodique que Montero, comédienne, nourrie de cette référence, passe insensiblement de l'allégorie, du gestus, à l'émotion conquise. On se posera alors la question suivante: en quoi ce détachement inabouti, cet accueil stylé de l'émotion ont-ils pu l'aider dans l'abord des songs de Mère Courage?

\section{Entre Vilar et Brecht : un tempérament de tragédienne}

Deux grandes figures du monde théâtral s'imposent dans son parcours: celle de Federico García Lorca (directeur artistique et auteur) et celle de Jean Vilar (directeur artistique et acteur). Lorca avec qui elle effectue ses premières tournées dans les zones rurales espagnoles, dont elle ne cessera d'interpréter les diverses adaptations françaises. Vilar avec qui elle collabore dès 1947 lors de la première Semaine d'art en Avignon, dont elle rejoint la troupe en 1951 dans la première aventure de Mère Courage à Suresnes. Sa fidélité au TNP se confirme lors de sa reprise du rôle-phare de Courage au Festival d'Avignon, en 1959 et en 1960.

\section{Comme une chanteuse dérobée au vedettariat}

Son parcours dans la chanson est plus éclectique que son parcours au théâtre, tant en termes de répertoire qu'en termes d'adresse au public. Elle commence par le cabaret, monte un récital "Germaine Montero " au Théâtre de l'Athénée en juin 1945, fait un passage à l'Olympia en 1956. Mais elle s'oriente bien davantage vers le disque, avec des

Revue Sciences/Lettres, 6 | 2019 
orchestres présents dans le studio d'enregistrement, et vers la radio pour des émissions où elle chante en direct, avec un simple accompagnement à la guitare ou à l'accordéon. Finalement on la vit peu sur scène dans le registre exclusif de chanteuse.

C'est en zone libre, avec Kosma au piano, qu'elle interprète Prévert dans divers cabarets de Nice, de Cannes, de Marseille. Devant un auditoire dérouté, le duo propose des titres inédits : Je suis comme je suis, Page d'écriture, En sortant de l'école... Elle s'est donc bien rodée comme chanteuse à l'épreuve du cabaret. Toutefois, elle ne profite pas vraiment de la période florissante de ces spectacles qui rencontrent l'engouement des artistes et des intellectuels à la Libération. Dès 1950, elle abandonne l'effervescence culturelle des petits lieux de la Rive gauche. Veut-elle aller vers des scènes plus vastes, des accompagnateurs attitrés ou des publics plus diversifiés?

Son tour de chant au Théâtre de l'Athénée en 1945 se compose de trente chansons mêlant la verve du goguettier Marc-Antoine Désaugiers, l'ironie de Prévert et la ferveur des mélodies populaires d'Espagne. Sa discographie témoigne elle aussi d'un audacieux éclatement entre Henri Michaux, Béranger, Xanrof, des refrains de Paris, des variétés madrilènes des années folles, des chansons de marins et des reprises de Fréhel, sans parler de ses auteurs fétiches. Le cadre du cabaret «Saint-Germain-des-Prés » ne lui aurait pas permis de vagabonder aussi aisément entre mémoire populaire chansonnière et chansons dites «d'avant-garde » ou "à texte ». Cette relative échappée du cabaret des années 1950 lui vaut sans doute de n'avoir jamais eu le statut de vedette comme une Cora Vaucaire, une Catherine Sauvage, une Patachou, voire une Juliette Gréco, qu'elle résume et annonce.

\section{S'étonner d'une rencontre}

41 Comment s'opère la rencontre entre Vilar et Montero ? Ce qui les rapproche : d'abord leur collaboration à la Semaine d'art en Avignon. Germaine Montero est une comédienne qui connaît un large succès d'estime. On sait que le rôle de Mère Courage a décidé de son ralliement à la troupe du TNP. Peut-être aussi une intuition musicale très sûre. Destinée à une carrière de chanteuse d'opéra, Germaine Montero est initiée dès son plus jeune âge au piano et au violon. Jean Vilar est musicien. Musicien instrumental et musicien du texte, sensible à la corporéité de la voix dans le jeu des acteurs. L'un et l'autre sont préoccupés par la voix des mots. Que ce soit sur disque ou sur scène, lui, si attentif à la musicalité du verbe, n'a pas pu manquer cette voix âpre, ce tissage entre délié de la diction et intensité dramatique mis au service de la chanson non seulement littéraire mais aussi populaire. Car ils ont peut-être aussi en commun un certain rapport de vision du populaire, de sensibilité au populaire: ni l'un ni l'autre ne sont «politiques » au sens partisan du terme ; ils ont par rapport au peuple une conviction empathique que Vilar sera amené à développer dans son action pour le TNP ${ }^{21}$, que Germaine Montero manifestera dans ses références artistiques.

\section{Chant contre chants}

En 1951, Vilar monte Mère Courage. La presse témoigne d'une réception critique. On reproche d'abord à Vilar d'avoir servi une "œuvre d'un Allemand de l'Est», une « œuvre communiste », qui « insulte l'armée ». Ce rejet est politique. La désapprobation esthétique, elle, fera suite aux représentations de la pièce en langue allemande, dans la mise en scène de Brecht et l'interprétation d'Helene Weigel en 1954. Un fossé se creuse 
entre l'adhésion populaire au travail de Vilar et de Montero et le rejet des brechtiens tout à leur engouement pour le Berliner Ensemble.

Cette polémique perdure. Citons Philippe du Vignal en 2014: «La mise en scène de Vilar était d'une grande intelligence mais les songs de Paul Dessau en français mal traduit [sic], ne valaient évidemment pas ceux du Berliner en allemand ${ }^{22} . . . »$. Sans juger ici de la traduction française des songs, il est indispensable de rappeler qu'une voix est principalement sculptée par l'apprentissage de sa langue initiale. Il est alors inutile de faire un parallèle entre Weigel et Montero du strict point de vue de la sonorité du chant, toujours situé dans le sillage d'une langue maternelle, de ses potentialités et matérialités phoniques, syllabiques, accentuelles, rythmiques, porteuses, en deçà de l'énonciation, de secousses émotives et de valeurs symboliques. Comme il existe un intraduisible du poème, il existe un intraduisible du chant d'une langue. Il faut en écoutant Montero faire le deuil de la langue allemande et de la façon dont celle-ci donne forme aux songs de Weigel.

\section{Double jeu}

Les critiques comparèrent les deux interprètes, au détriment d'une Montero à qui aurait manqué «la formation brechtienne». À entendre et à regarder les deux interprétations, ce qui frappe chez cette dernière, c'est non pas une mimétique approximative mais un écart manifeste en matière de théatralisation de la voix chantée. À ne prendre que la chanson d'ouverture dite Chanson de Mère Courage, ce qui s'impose du côté de Germaine Montero, c'est la force, l'énergie, la fermeté du placement vocal. Sur cette marche, sa voix est altière; la prononciation de chaque syllabe est découpée, martelée; le phrasé souvent ponctué de « $\mathrm{r}$ » roulés trace son sillon avec assurance et non sans dureté. L'archive visuelle montre Germaine Montero dans une posture très droite, avec un visage grave. Sur la note ascendante finale, elle lève la main en direction du public qui l'applaudit. Autrement dit, venue à la chanson par le chemin du théâtre, elle ne peut cependant se départir de son métier de chanteuse. Elle soigne son vibrato et retrouve sa gestuelle d'adresse à ceux qui sont venus l'entendre. Dans ce contrepoint du song, le langage de la chanson est impératif, il prend le pas sur le langage du jeu théâtral. Il en va tout autrement pour Weigel qui chante avec entrain, d'une voix sûre, mais dans la seule logique du rôle de Courage, désinvolte en ce début d'épopée puisque « les affaires » tournent bien. Weigel n'est pas sur le registre du double jeu: s'affirmer en chanteuse, s'affirmer en actrice. Nous ne sommes pas, entre France et Allemagne, dans la même histoire de la scène chansonnière ; entre Weigel et Montero, le parcours artistique n'a pas été le même.

\section{Morphologie musicale}

Pour parler des songs comme d'éléments didactiques, appuis récitatifs au regard de l'action et des dialogues des personnages, et donc comme accélérateurs du gestus social, encore faut-il les placer dans la structure musicale d'ensemble de la pièce. Si l'on se réfère au disque intitulé Germaine Montero chante Mère Courage, l'élément musical dure trente-huit minutes pour un spectacle de deux heures environ. La Chanson de Mère Courage revient quatre fois en voix chantée et quatre fois en version instrumentale. À ce fil rouge lancinant de la marche s'ajoutent sept autres songs: quatre en voix solo féminine (trois pour Mère Courage, une pour Yvette), une seule en voix solo masculine. 
Restent deux songs chantés en duo. Sur le découpage en douze tableaux, la chanson, selon une intensité variable, parcourt toutes les scènes. Pour apprécier la spécificité vocale de Germaine Montero, il faut mesurer la grande différence mélodique entre ces différents songs. La Chanson de Mère Courage, la Chanson de la rose, la Berceuse suivent une composition très classique, sans rupture de ton ou de style. Il en va tout autrement des deux chansons centrales à voix féminine soliste: la Chanson de la fraternisation et la Chanson de la grande capitulation qui suivent une ligne de déchirure des euphonies.

\section{Chanter pour dire}

La présence vocale de Montero est à apprécier du point de vue de son adaptation sans faille aux divers styles de compositions musicales :

- Dans le leitmotiv de Mère Courage, la diction de Montero est implacable. Les accents de véhémence, les points d'exclamation de son chant semblent - chose rare - faire entendre les ponctuations de l'écrit dans l'oral. Dans tous les retours de la Chanson de Mère Courage, évoquée plus haut, Montero maintient la même stature de puissance, défiant le sort, dans sa voix chantée et dans le caractère dramatique de son rôle ; alors que Weigel, qui chantait de façon enjouée au début de la pièce, change de registre au fur et à mesure du déroulement de son infortune ; in fine, sa voix est lasse, souffrante.

- Dans les chansons a cappella, Germaine Montero retrouve toutes les nuances lyriques qu'elle a pu apporter aux chansons littéraires ou plus simplement sentimentales qu'elle chante avec délicatesse. Tandis que pour le dernier song intitulé Berceuse, Weigel chantonne, parle de façon presque inaudible, de façon très émouvante, le visage penché, le corps totalement replié sur le corps allongé de Catherine, sa fille morte.

- L'alternance du parler et du chant dans la Chanson de la grande capitulation est un morceau de bravoure. Qu'entend-on ? Un refrain à mélodie mémorisable au tempo lent, une accélération du tempo, un changement mélodique très accentué, une ligne mélodique plate et hachée, une intrusion subite du parler sur l'énoncé de paroles ayant l'allure de proverbes, puis un retour au refrain presque mélodieux. Montero enchaîne cela sans aucune hésitation, avec une fluidité accordée à la trame instrumentale. Il fallait l'extrême plasticité de sa voix pour réussir sans dissonance un tel exercice. Brecht souhaitait que la chanteuse s'écoute chanter, ce que ne fait pas Montero, son assurance n'a pas besoin de cela. Reste que pour chanter, il faut tout de même s'entendre chanter, et Germaine Montero ne peut pas négliger l'attention à la justesse de sa voix. Sans doute touche-t-on là une contradiction sociale fondamentale entre un goût populaire franc pour la chanson et un refus intellectualiste d'assumer ce goût dans toute sa force naïve, heureuse et puissante.

L'écoute de l'enregistrement de 1957 à Chaillot nous donne un indice de ce fossé historique des cultures. D'abord le public est très réactif, on entend beaucoup de rires ; les répliques de Mère Courage au Capitaine, au Recruteur, à l'Aumônier, font mouche ; on entend aussi beaucoup d'applaudissements. Ils suivent régulièrement la fin de chaque insert chanté. Autrement dit, si l'on rit d'un bon mot, on salue, comme au récital, la performance technique et esthétique de la voix chantée. Comme si s'alliaient en ce même espace deux registres de réceptivité : la distance du rire pour le jeu parlé, l'implication aimante pour le jeu chanté.

On notera d'ailleurs que, pour partie, les thèmes des chansons de Mère Courage rappellent ceux de la «chanson néoréaliste » appelant au Destin (« L'homme propose, Dieu dispose »), à la complicité entre le prédateur et la proie, et que nombre de ces 
paroles sonnent comme des proverbes et dictons. Tous les signes sont là pour reconnaître la complainte dont le spectateur est potentiellement l'auditeur familier. Pas de place pour une didactique de la chanson, pour un espace critique, si ce n'est dans la perspective d'une déconstruction coupée de toute racine populaire.

Les chansons ne sont pas raisonnables, elles ont leur vie propre. Pour apprécier la contradiction entre mission dévolue aux songs et insouciance de belle échappée, l'exemple de L'Opéra de quat'sous est encore plus frappant. En effet, les compositions de Kurt Weill sont pensées dans cette logique d'éloignement de la seule vertu émotionnelle de la chanson. Or, même si les chansons parodiques telles que la Complainte de Mackie le surineur, le Chant d'adieu de Polly, la Chanson de Jenny ne sont pas des chansons faciles, elles vont pourtant se retrouver sur toutes les lèvres.

Il s'agissait selon l'ambition initiale de transfigurer la rengaine, d'en faire le véhicule d'une idée sans en abolir le pouvoir attractif. Sur ce dernier point la réussite dépasse l'espérance, mais seul le bonheur de chanter subsiste effaçant toute intentionnalité critique. La Complainte de Mackie absorbée par la force épique des voix d'Armstrong, d'Ella Fitzgerald, le charme de Frank Sinatra, devient un standard international, voyage dans l'imaginaire sentimental des foules, détachée de toute référence à une nouvelle dramaturgie rééducatrice.

Et revenant à la Mère Courage du TNP, sans doute faut-il en ce sens insister sur la configuration d'une écoute française de la pièce qui, hors orthodoxie théâtrale, s'était, via le disque et le concert, formée à la plainte des " grandes réalistes » devenues voix éponymes des chansons.

[Lien au dossier pédagogique multimédia en ligne « Entendre le théâtre », à venir début juillet 2019]

\section{Rosy ou la voix face au rire désenchanteur}

Rosy Varte est une actrice formée au cabaret ; sa carrière est déjà bien assise lorsqu'elle rejoint Jean Vilar qui, en 1957, s'engage dans le montage des différents Ubu d'Alfred Jarry pour un spectacle du TNP et la distribue dans le rôle de la Mère Ubu. Cette farce gentiment « anarchisante » ne semble pas a priori disposée à accueillir la chanson, si ce n'est pour la tourner en dérision. On sait que Jarry fréquentait le Chat noir d'Aristide Bruant, père de la « chanson des apaches » dite aussi « chanson de barrière ».

Cependant le spectacle est bel et bien scandé par des impromptus musicaux, le plus souvent instrumentaux (accordéon, orgue de barbarie). Il comporte douze chansons, dont on peut entendre la version discographique, enregistrée en studio pour la série "spéciale TNP ${ }^{23}$.

54 La Chanson du décervelage se présente comme une véritable charge de la chanson réaliste, incluant dans son sarcasme tout à la fois la ligne mélodique, le grain vocal, l'instrumental, la stéréotypie thématique et lexicale. Cette mise en voix de figures ouvrières (l'ébéniste), populaires (la modiste), campées lors de leur sortie dominicale, engoncées tant dans leurs « beaux accoutrements » que dans leurs parlures argotiques, s'amuse de la sottise ordinaire des gens du commun. Reste la question de l'interprétation qui peut faire glisser la dérision vers le plaisir et contourner ainsi le désamour de la chanson via cette distance burlesque si habilement soulignée. 

théâtralisation de la parole chantée : différence des modes d'initiation au voyage dans le chant, différence des styles d'adresse au public, différence des émotions mobilisées dans l'expression et dans la réception, différence des investissements de carrière et de vie. un mode partagé de volonté de puissance de la voix, de volonté de convaincre par la justesse et la précision du phrasé ; l'acquisition, dans tous les cas, d'une maitrise technique venue étayer un don naturel, d'une maitrise sémantique des symbolismes vocaux; enfin la marque, pour chacune des chanteuses, d'une forte singularité de timbre et/ou de placement de la voix dans un ambitus assez étendu.

Cela peut certes apparaître comme des traits trop généraux. Toutefois, au regard de l'histoire du spectacle et de l'histoire de la chanson, l'exposition de la voix, le répertoire spécifique, la signature vocale unique, le sens et le savoir musical dans la conduite du souffle peuvent bien désormais figurer comme appartenant au même paysage d'un passé révolu. Le temps qui passe les rapproche. La mise aux normes des sonorisations, la mode des voix faibles, lisses, interchangeables, l'uniformisation des productions balayant le poids des compositions et des mots, tout cet effacement de 
l'esthétique originale des chansons souligne avec le recul leur appartenance commune à un monde-conservatoire de la langue et de la vitalité psychique, sociale, mémorielle de la chanson.

Même si Piaf s'inscrit dans la forme pleine du chant profond, dans le rituel stabilisé du récital, là où ne vont pas Montero et Varte, entraînées vers des langages scéniques plus expérimentaux, il reste que la partition chantée de Courage par Montero résiste à l'inversion souhaitée de la valeur enthousiaste du chant et que Rosy Varte triomphe de la profanation attendue. Qu'y a-t-il de plus éloigné d'un Jarry cherchant à corroder sous un rire acide aussi bien les grands tragiques que la tradition de la chanson populaire, que la traversée mystique d'une Piaf, tout effort voué à l'âme douloureuse des chansons? Le malentendu n'est-il pas moins grand entre un Brecht qui considère la rengaine comme un opium du peuple et une Germaine Montero à la recherche des traditions orales flamenca les plus authentiques?

61 Au bout du compte, dans ce moment français de conquête de l'art chansonnier par la scène, peu importe les prescripteurs de transgression, le langage des chansons sait encore imposer la marque de son espace vibrant, indomptable.

\section{NOTES}

1. Le Bel Indifférent, pièce en un acte (un monologue) de Jean Cocteau, a été joué entre le 20 et le 25 avril 1940 au théâtre des Bouffes-Parisiens par Édith Piaf et Paul Meurisse (rôle muet).

2. Yvette Guilbert, L'Art de chanter une chanson, Paris, Grasset, 1928, p. 145.

3. Expression de Michel de Certeau, La Culture au pluriel, Paris, Seuil, [1974] 1993.

4. Du point de vue de la diversité de la langue française, la chanson est aussi un conservatoire de sociolectes et de dialectes comme en témoignent notamment Gaston Couté ou Jules Jouy. Bruant renoue avec l'argot des coquillards, celui de Villon. La tradition sera reprise par Brassens.

5. Roman Jakobson, Six leçons sur le son et le sens, Paris, Minuit, 1976.

6. De nombreuses définitions de la chanson française vont la caractériser par son lien aux formes classiques de la mélodie française, par une ligne d'ensemble toujours subordonnée à l'intelligibilité du texte.

7. Bien qu'accordant une place prépondérante à la voix des chansons, la musicologie récente ne se départit pas de son formalisme. Voir Céline Chabot-Canet, «Entre universalité et singularité. L'interprétation vocale abordée par l'outil informatique ", Conférence audiovisuelle, IRCAM, décembre 2014.

8. Joëlle-Andrée Deniot, Les Ouvriers des chansons (www.chansons-francaises.info).

9. Les nouvelles technologies du son remettent en cause ce primat de l'empreinte vocale unique. Pour conserver une singularité, certains chanteurs adoptent des tics vocaux. Par exemple, l'attaque dans le souffle permet à Julien Doré d'être reconnu à la première syllabe.

10. Joëlle-Andrée Deniot, Édith Piaf, la voix, le geste, l'icône. Esquisse anthropologique, Paris, Lelivredart, 2012.

11. Voir Mon légionnaire, L'Accordéoniste, Je t'ai dans la peau, Le Temps des forains, Le Gitan et la fille, Mon Dieu, Le Clown, L'Hymne à l'amour, etc. 
12. Certaines prestations de Brel peuvent aller à l'encontre de cet argument. Pourtant Mon Dieu reste du point de vue développé plus fort que Ne me quitte pas.

13. Joëlle-Andrée Deniot, "Les chansons de la vie en noir ", in Alain Pessin et Marie-Caroline Vanbremeersch (dir.), Les Euvres noires de l'art et de la littérature, t. 2, Paris, L'Harmattan, 2002, p. 159-184.

14. Contrairement à ce qui est souvent affirmé, Piaf emploie peu de formes argotiques; elle ne joue sur ce style que dans ses tout premiers enregistrements et se règle au contraire sur un usage orthodoxe de la langue.

15. Helene Weigel, comédienne née à Vienne. Épouse de Bertolt Brecht. A interprété le rôle de Mère Courage à la reprise du spectacle en 1949.

16. Film de Marcel Carné de 1946. Jean Vilar y apparaît dans le rôle du Destin.

17. Propos tenus par Pierre Mac Orlan.

18. Du gris, Bénech/Dumont, 1931.

19. Définition de la tragédie dans la Poétique d'Aristote.

20. Terme de l'art pictural désignant la graduation subtile qui permet sans rupture franche de formes et de couleurs de passer d'un morceau, d'un pli, d'un motif d'étoffe à un autre, de la part d'ombre à la part de lumière reflétée dans le paysage, sur les visages et les objets.

21. Joëlle-Andrée Deniot, "Esthétique et médiation: oublier Vilar", in Gilles Ferréol (dir.), Médiations et régulations, Bruxelles, Éditions E.M.E., 2016, p. 237- 250.

22. Théâtre du blog, texte en ligne : http://theatredublog.unblog.fr/2014/09/19/mere-couragede-brecht/

23. Musique et chansons pour Ubu d'Alfred Jarry, disque 33 t. 1/3, HF, 17 cm, Véga, T.N.P. 2, 1959.

\section{RÉSUMÉS}

Édith Piaf, Germaine Montero, Rosy Varte : trois femmes proches en matière de génération, trois femmes qui laissent leur nom dans le monde de l'art et du spectacle; trois femmes qui chantent, dans des registres socio-esthétiques fort éloignés. Piaf est une icône de la chanson. Montero se définit d'abord comme comédienne. Varte débute par le cabaret. Au regard de ces divergences, toute approche transversale est compromise. Toutefois, le passage par la notion d'interprétation chansonnière, et par un double rapprochement historique et théorique entre théatre de la chanson et chansons du théâtre, nous permet de suivre le fil des nuances, des contrastes, voire des contradictions entre ces trois modes très singularisés d'occupation de l'espace théâtral du chant. On parlera de forme sanctuarisée dans le contexte du récital pour Édith Piaf, de forme réitérative/combative du chanter pour Germaine Montero, voix française de Mère Courage, de forme brève et corrosive pour Rosy Varte dans Ubu roi.

Edith Piaf, Germaine Montero, Rosy Varte: three women close in age, three women who made their mark on the world of art and performance; three women who sung in radically different socio-aesthetic vocal registers. Piaf is a singing icon. Montero first defined herself as an actress. Varte got her start in cabaret. Given these differences, any transversal approach is compromised. However, by using the notion of song interpretation and a dual historical and theoretical rapprochement between the theatre of song and the songs of the theatre, we follow the thread of nuances, of contrasts, of contradictions, even, between these three highly singular modes of occupying the theatrical space of song - namely the enshrined form in the context of the recital 
for Edith Piaf, the reiterative/combative form for Germaine Montero (the French voice of Mother Courage), and the brief and corrosive form for Rosy Varte in Ubu Roi.

\section{INDEX}

Mots-clés : Édith Piaf, Germaine Montero, Rosy Varte, interprétation, théâtralité, chanson, voix, récital

Keywords : Edith Piaf, Germaine Montero, Rosy Varte, interpretation, theatricality, song, voice, recital

\section{AUTEUR}

\section{JOËLLE DENIOT}

Joëlle-Andrée Deniot, professeur émérite de sociologie à Nantes, rattachée à l'UE C3S de l'Université de Franche-Comté, est spécialisée en socio-anthropologie du langage, de l'esthétique, des cultures populaires, de la voix et des chansons. Elle a en particulier publié de nombreux articles consacrés à la chanson réaliste et à la voix chantée.

Parmi ses publications :

Édith Piaf, la voix, le geste, l'icône, Paris, Lelivredart, 2012.

Le Genre et l'effroi d'après Judith Butler, Nantes, Cahiers du Lestamp, 2013.

Eros et liberté, avec A. Mouchtouris et J. Réault, Paris, Le Manuscrit, 2014.

Le Sentiment esthétique. Essai transdisciplinaire, Paris, Le Manuscrit, 2017. 